\title{
MODELING OF WELD POOL BEHAVIOUR IN SPOT WELDING BY PULSED LASER RADIATION*
}

\author{
A.P. SEMYONOV, I.V. SHUBA, I.V. KRIVTSUN and V.F. DEMCHENKO \\ E.O. Paton Electric Welding Institute, NASU \\ 11 Bozhenko Str., 03680, Kiev, Ukraine. E-mail: office@paton.kiev.ua
}

\begin{abstract}
Process of metal evaporation in laser welding is accompanied by dynamic impact of metal vapours on pool free surface. As this process proceeds non-uniformly over pool surface, reactive vapour pressure can differ considerably in its different sections, leading to depression of melt free surface. This work proposes a mathematical model allowing investigation of penetration dynamics and shape of weld pool free surface in laser welding of sheet metals by a stationary pulsed source (spot welding). When developing the model, we assumed that heat transfer process in the metal is due to heat conductivity and convection, and heat losses from the surface are due to metal evaporation and energy loss for heat radiation. The work gives the results of numerical modeling of weld pool penetration dynamics derived with application of the developed model. 6 Ref., 7 Figures.
\end{abstract}

Keywords: laser spot welding, sheet material, mathematical model, penetration dynamics, weld pool shape

Impact of concentrated energy sources, such as focused laser beam, on metal products being welded, may lead to intensive evaporation of metal from weld pool surface. Evaporation process is accompanied both by heat losses and dynamic impact of metal vapours on melt free surface. Under certain heating conditions, reactive vapour pressure can be quite high to cause significant depression of weld pool surface. Moreover, heating by a concentrated energy source creates a high temperature gradient along the melt free surface that is the cause for generation of $\mathrm{Ma}^{-}$ rangoni thermocapillary convection in weld pool volume. As shown by previous studies [1], thermocapillary surface forces have an essential influence on the pattern of metal flow in the weld pool and largely determine the dimensions and shape of penetration zone in different fusion welding processes.

This study proposes a mathematical model describing thermal and hydrodynamic processes in molten metal pool in spot laser welding of sheet metal. For model development it was assumed that heat transfer in the metal occurs through heat conductivity and convection, whereas heat losses from the surface are due to metal evaporation and heat radiation flow. Hydrodynamic process model used in the work is based on

\footnotetext{
${ }^{*}$ Basing on the paper presented at the Int. Conf. on Laser Technologies in Welding and Materials Processing (27-31 May 2013, Katsively, Crimea, Ukraine).
}

(๑ A.P. SEMYONOV, I.V. SHUBA, I.V. KRIVTSUN and V.F. DEMCHENKO, 2014
Navier-Stokes equations for viscous incompressible liquid and allows for capillary pressure due to curvature of molten metal free surface, as well as Marangoni thermocapillary effect. For model simplification it was assumed that all the described physical processes have the property of axial symmetry. The work analyzes the influence of various physical factors on penetration process dynamics. For this purpose comparative analysis of three models of weld pool dynamics was performed:

- heat conductivity model;

- model of convective-conductive heat transfer allowing for free surface curvature due to reactive vapour pressure;

- model of convective-conductive heat transfer allowing for reactive vapour pressure and thermocapillary Marangoni effect.

Assessment of metal movement speed. Before describing the mathematical model, let us assess the speed of melt motion in the weld pool in spot laser welding of sheet low-carbon steel. Material properties used in further calculations, are taken from work [2] and are given below:

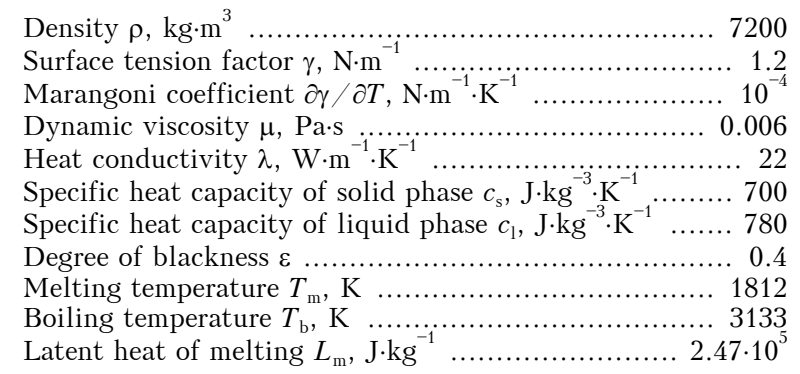

Let us write an approximate expression for tangential stresses $P_{\mathrm{m}}$ on molten metal surface $\Gamma$, assuming that the surface proper is flat: 


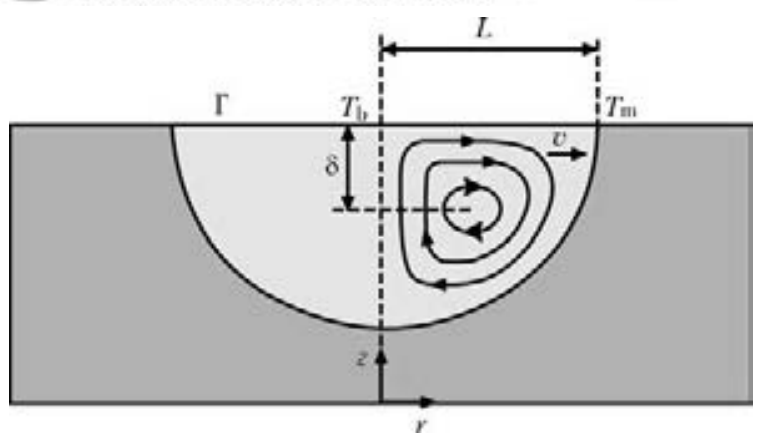

Figure 1. Characteristic flow pattern in the weld pool at thermocapillary Marangoni convection

$$
P_{\mathrm{m}}=\left.\mu \frac{\partial u}{\partial z}\right|_{\Gamma} \approx \mu \frac{u_{\mathrm{s}}}{\delta},
$$

where $\mu$ is the dynamic viscosity of liquid metal; $u_{\mathrm{s}}$ is the value of radial component of speed vector on the surface; $\delta$ is the distance from the surface to eddy center (Figure 1).

On the other hand, for value $P_{\mathrm{m}}$ we can write

$$
P_{\mathrm{m}}=\left.\frac{\partial \gamma}{\partial T} \frac{\partial T}{\partial r}\right|_{\Gamma} \approx \frac{\partial \gamma}{\partial T} \frac{T_{\mathrm{b}}-T_{\mathrm{m}}}{L},
$$

where $\gamma$ is the surface tension coefficient of liquid metal; $L$ is the weld pool radius. At derivation of (2) we assumed that temperature in the pool center corresponds to boiling point under normal conditions. We will also assume $\delta=L / 4$, then we will finally have

$$
u_{\mathrm{s}}=\frac{\delta}{\mu L} \frac{\partial \gamma}{\partial T}\left(T_{\mathrm{m}}-T_{\mathrm{b}}\right) \approx 5.5 \mathrm{~m} / \mathrm{s} .
$$

Value of Peclet number for the considered conditions $\mathrm{Pe}=u_{\mathrm{s}} L / \alpha=630$, where $\alpha$ is the coefficient of molten metal heat conductivity, indicates that convective heat transfer will have an essential influence on metal pool heat condition.

Description of mathematical model. Numerical solution procedure. To describe thermal processes in the metal being welded, we will use the equation of heat transfer in area $\Omega$ (Figure 2):

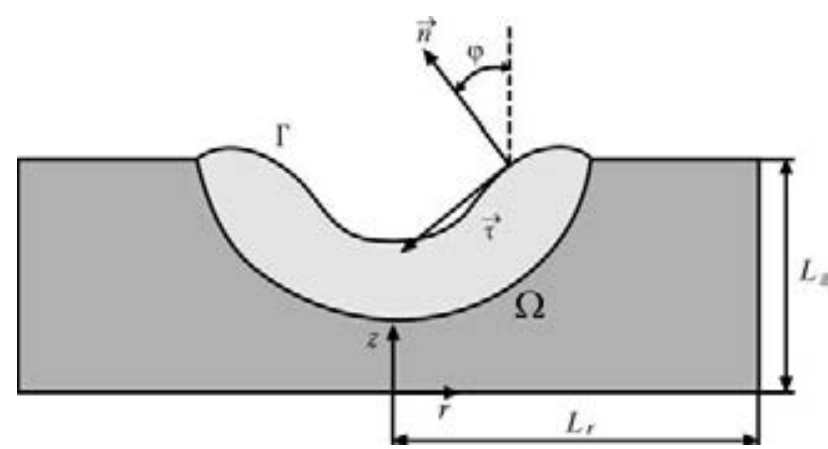

Figure 2. Schematic of calculated area

$$
\begin{gathered}
\frac{\partial H}{\partial t}+u \frac{\partial H}{\partial r}+v \frac{\partial H}{\partial z}=\frac{1}{r} \frac{\partial}{\partial r} \times \\
\times\left(r \lambda \frac{\partial T}{\partial r}\right)+\frac{\partial}{\partial z}\left(\lambda \frac{\partial T}{\partial z}\right), \\
H(T)=\int_{T_{0}}^{T} c \rho d \bar{T}+L_{\mathrm{m}} \rho \eta,
\end{gathered}
$$

where $u, v$ are the components of speed vector; $H$ is the specific enthalpy; $\eta$ is the liquid phase fraction. We will complement equation (4) by boundary and initial conditions:

$$
\begin{gathered}
\lambda \frac{\partial}{\partial} \mid=A\left(T_{\Gamma}, \varphi\right) \cos (\varphi) q_{\mathrm{S}}(r)- \\
-q_{\mathrm{ev}}\left(T_{\Gamma}\right)-q_{\mathrm{rad}}\left(T_{\Gamma}\right), \\
\left.\frac{\partial T}{\partial z}\right|_{z=0}=\left.\frac{\partial T}{\partial r}\right|_{r=0}=\left.\frac{\partial T}{\partial r}\right|_{r=L_{r}}=0, \\
T(r, z, 0)=T_{0}, \quad(r, z) \in \Omega .
\end{gathered}
$$

The following designations were introduced in condition (6): $A$ is the coefficient of laser radiation absorption by metal surface; $q_{\mathrm{s}}$ is the laser radiation power density; $q_{\mathrm{ev}}$ is the specific heat flow due to metal evaporation from pool surface; $q_{\text {rad }}$ is the specific radiant heat flow. To describe radiation power distribution in the laser beam the following dependence was used:

$$
q_{\mathrm{s}}(r)=q_{0} \exp \left(-2\left(r / r_{0}\right)^{2}\right),
$$

where $q_{0}$ is the intensity of laser radiation on beam axis; $r_{0}$ is the beam radius on item surface. At calculation of absorption coefficient it was assumed that laser radiation has random polarization. In this case

$$
A\left(T_{\Gamma}, \varphi\right)=1-\frac{R_{s}\left(T_{\Gamma}, \varphi\right)+R_{p}\left(T_{\Gamma}, \varphi\right)}{2} .
$$

Here

$$
\begin{gathered}
R_{s}\left(T_{\Gamma}, \varphi\right)=\left|\frac{\cos \varphi-\sqrt{\varepsilon_{\omega}\left(T_{\Gamma}\right)-\sin ^{2} \varphi}}{\cos \varphi+\sqrt{\varepsilon_{\omega}\left(T_{\Gamma}\right)-\sin ^{2} \varphi}}\right|^{2}, \\
R_{p}\left(T_{\Gamma}, \varphi\right)=\left|\frac{\varepsilon_{\omega}\left(T_{\Gamma}\right) \cos \varphi-\sqrt{\varepsilon_{\omega}\left(T_{\Gamma}\right)-\sin ^{2} \varphi}}{\varepsilon_{\omega}\left(T_{\Gamma}\right) \cos \varphi+\sqrt{\varepsilon_{\omega}\left(T_{\Gamma}\right)-\sin ^{2} \varphi}}\right|^{2}
\end{gathered}
$$

are the coefficients of reflection of $s$ - and $p$-polarized waves by metal surface, respectively; $\varphi$ is the angle of incidence (see Figure 2); $\varepsilon_{\omega}$ is the complex dielectric permeability of metal at laser radiation frequency. Energy losses due to heat radiation were found from Stefan-Boltzmann law: 


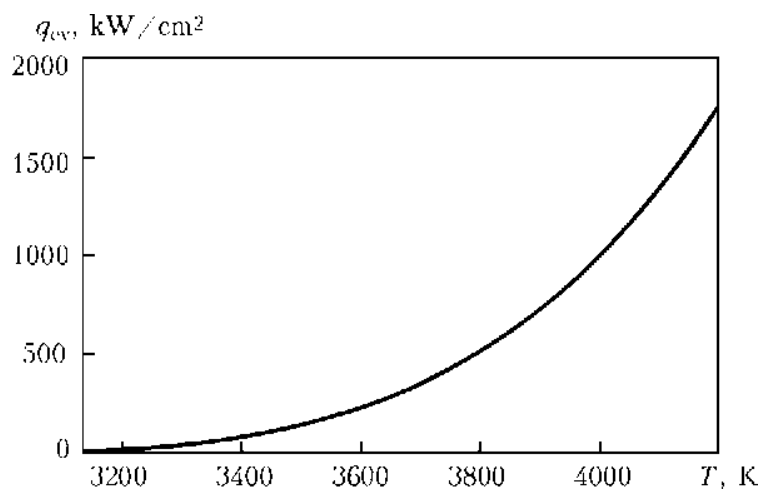

Figure 3. Temperature dependence of heat losses through evaporation

$$
q_{\mathrm{rad}}\left(T_{\Gamma}\right)=\varepsilon \sigma\left(T_{\Gamma}^{4}-T_{\infty}^{4}\right),
$$

where $\sigma$ is the Stefan-Boltzmann constant; $T_{\infty}$ is the ambient temperature.

Model of hydrodynamic processes in the weld pool is based on Navier-Stokes equations for viscous incompressible liquid:

$$
\begin{gathered}
\rho\left(\frac{\partial u}{\partial t}+u \frac{\partial u}{\partial r}+v \frac{\partial u}{\partial z}\right)=\frac{1}{r} \frac{\partial}{\partial r}\left(r \sigma_{r r}\right)+ \\
+\frac{\partial \sigma_{r z}}{\partial z}-\frac{\sigma_{\varphi \varphi}}{r}, \\
\rho\left(\frac{\partial v}{\partial t}+u \frac{\partial v}{\partial r}+v \frac{\partial v}{\partial z}\right)=\frac{1}{r} \frac{\partial}{\partial r}\left(r \sigma_{r z}\right)+ \\
+\frac{\partial \sigma_{z z}}{\partial z}-g \rho, \\
\frac{1}{r} \frac{\partial}{\partial r}(r u)+\frac{\partial v}{\partial z}=0,
\end{gathered}
$$

where

$$
\begin{gathered}
\sigma_{r r}=-P+2 \mu \frac{\partial u}{\partial r} ; \quad \sigma_{r z}=\mu\left(\frac{\partial v}{\partial r}+\frac{\partial u}{\partial z}\right) ; \\
\sigma_{z z}=-P+2 \mu \frac{\partial v}{\partial z}
\end{gathered}
$$

$\sigma_{\varphi \varphi}=-P+2 \mu \frac{u}{r}$ are the $\tilde{\sigma}$ stress tensor components; $g$ is the free fall acceleration; $P$ is the pressure in the melt. We will write boundary conditions for equations (11)-(13) in the following form:

$$
\begin{gathered}
\tilde{\sigma} \vec{n}=-\left(2 \gamma K+P_{\mathrm{ev}}\right) \vec{n}+\frac{\partial \gamma}{\partial s} \overrightarrow{\tau,} \\
\left(\vec{n},\left.\vec{V}\right|_{\Gamma}\right)=\left(\vec{n}, \vec{V}_{\Gamma}\right), \\
\left.\vec{V}\right|_{r=L_{r}}=\left.\vec{V}\right|_{r=0}=\left.\vec{V}\right|_{z=0}=0,
\end{gathered}
$$

where $\vec{n}$ and $\vec{\tau}$ are the unit vectors of normal and tangential to the surface, respectively (see Figu-

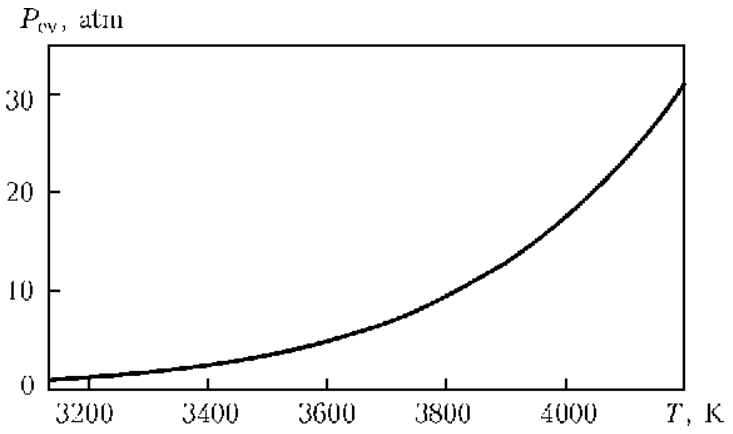

Figure 4. Temperature dependence of reactive pressure of metal vapours

re 2); $K$ is the average curvature of liquid metal surface; $\vec{V}_{\Gamma}$ is the speed of boundary movement. Equation (14) describes the balance of normal and tangential stresses on the pool free surface, whereas (15) gives the kinematic boundary conditions.

To solve problems (4)-(8) and (11)-(16) arbitrary Lagrangian-Eulerian method [3] was applied together with finite element method [4]. Calculated area $\Omega$ (see Figure 2) covers liquid metal zone, and also includes the solid phase. When solving the hydrodynamic problem, viscosity in the solid phase is assumed to be high enough $\left(10^{8}\right.$ higher than liquid metal viscosity $)$. During performance of calculation experiment, it allows suppressing metal movement in the solid phase, and eliminates the need to adapt the FE net for melting front shape and assign additional boundary conditions in the melting front. Such an approach was proposed for the first time in [5], and is found in publications under the title «effective viscosity method».

Numerical modeling results. Numerical experiments were conducted for the conditions of laser spot welding (Nd:YAG-laser) of low-carbon steel $0.3 \mathrm{~mm}$ thick. Temperature dependencies of evaporation process characteristics (heat losses through evaporation and reactive pressure of met-

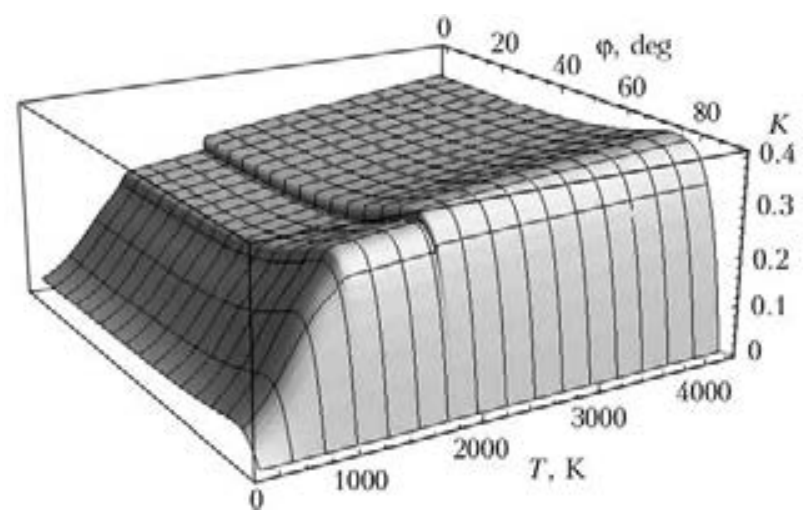

Figure 5. Dependence of coefficient of absorption of laser radiation (1.06 $\mu \mathrm{m}$ wave length) by the surface of low-carbon steel on temperature and angle of incidence 

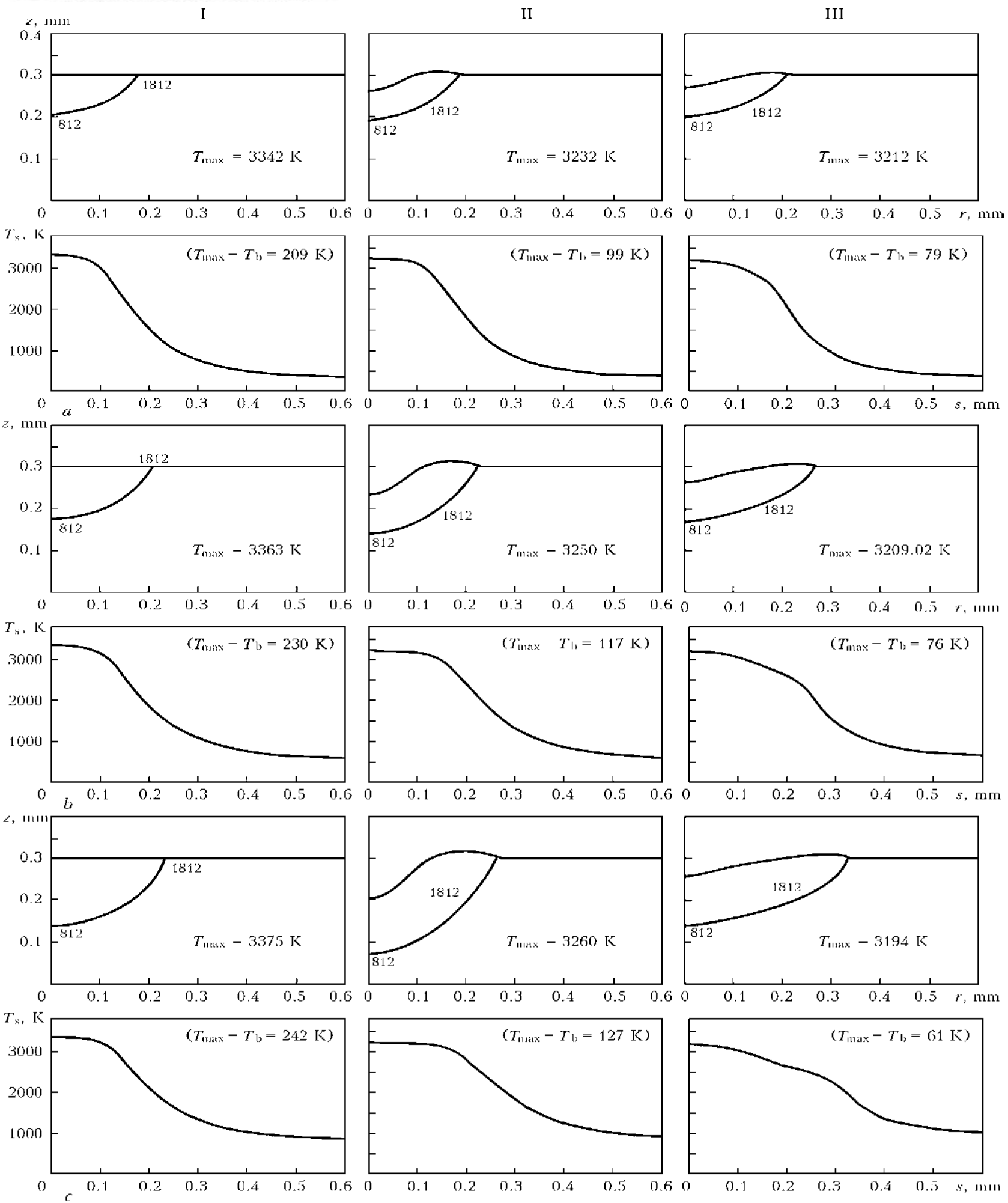

Figure 6. Dynamics of change in time $t$ of weld pool shape and temperature distribution along melt surface for modes I-III: $a-t=0.01 ; b-0.02 ; c-0.03 \mathrm{~s}$

al vapours) were derived using a model proposed in [6], and are given in Figures 3 and 4. Dependence of the coefficient of absorption for laser radiation with selected wave length on angle of incidence and metal surface temperature, determined by expression (10), is given in Figure 5 . Process parameters, used in calculations, are given below:
Welded metal thickness, $\mathrm{mm}$ 0.3 Maximum intensity of laser radiation on metal surface, $\mathrm{W} \cdot \mathrm{cm}^{-2}$

Laser beam radius on metal surface, $\mathrm{mm} \ldots \ldots \ldots . . . . .0 .2$

As shown by the results of numerical studies, calculated values of surface temperature in the center of laser heating spot, generated by model I, greatly increase boiling temperature ( $F i-$ 
1

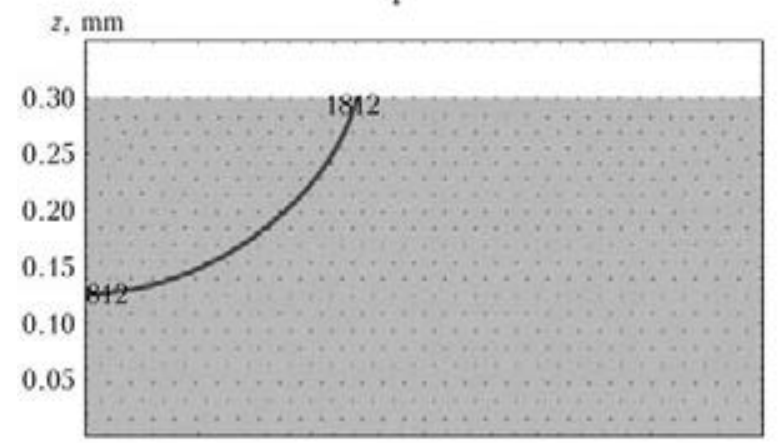

II

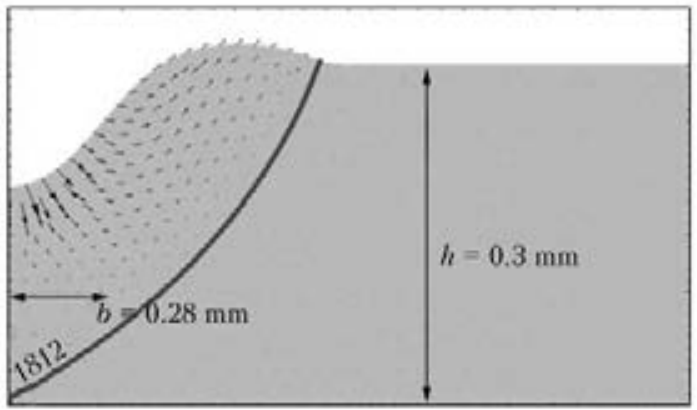

III

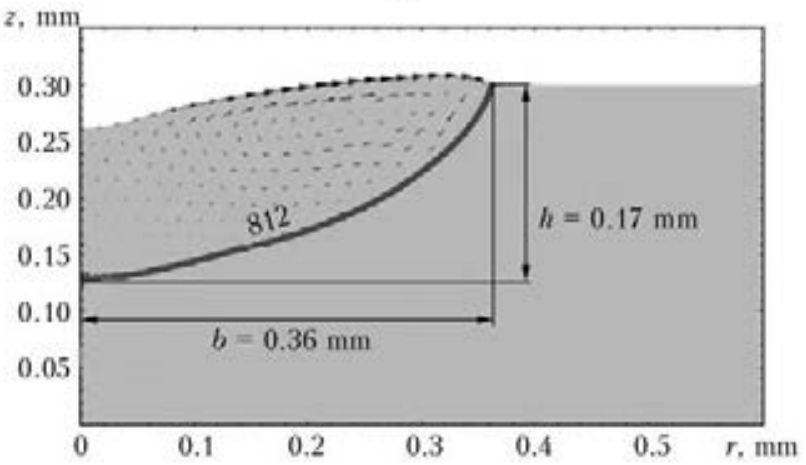

Figure 7. Comparative analysis of dimensions and shape of penetration zone produced using models I-III at $t=0.0332 \mathrm{~s}$ and maximum speed of 0 (I), 0.00285 (II) and 1.264 (III) $\mathrm{m} / \mathrm{s}$

gure 6). The smallest overheating of the surface above boiling temperature is observed with application of model III. Weld pool dimensions and shape differ accordingly. So, at one and the same time of laser radiation impact on welded metal surface $(t=0.0332 \mathrm{~s})$ depth $h$ and half-width $b$ of the penetration zone are equal to 0.17 and $0.24 \mathrm{~mm}$ for model I, 0.30 and $0.28 \mathrm{~mm}$ for model II, and 0.17 and $0.36 \mathrm{~mm}$ for model III, respectively (Figure 7).

On the whole, modeling results given in $\mathrm{Fi}^{-}$ gures 6 and 7 allow stating that Marangoni convection mainly influences the width of penetration zone, whereas pool surface depression promotes an increase of its depth. This results from increase of heat flow to the solid phase, due to reduction of thickness of liquid metal interlayer between pool surface and melting boundary.

Thus, the most adequate mathematical model of metal penetration in spot laser welding of sheet materials is that of convective-conductive heat transfer in the weld pool, allowing for reactive pressure of vapours on its surface and thermocapillary Marangoni effect (model III). This model allows in the most comprehensive manner for the processes of heat and mass transfer on the surface and in the volume of weld pool in laser welding of thin metal.

1. Kou, S. (2012) Fluid flow and solidification in welding: Three decades of fundamental research at the university of Wisconsin. Welding J., 91, 11 .

2. Hu, J., Tsai, H.L. (2007) Heat and mass transfer in gas metal arc welding. Pt 1: The arc. Int. J. Heat and Mass Transfer, 50, 833-846.

3. Donea, J., Huerta, A., Ponthot, J.Ph. et al. (2004) Arbitrary Lagrangian-Eulerian methods: Encyclop. of computational mechanics. Vol. 1. John Wiley \& Sons.

4. Zienkiewicz, O.C., Taylor, R.L. (2000) The finite element method. Vol. 1: The basis. Oxford: Butterworth-Heinemann

5. Kou, S., Sun, D.K. (1985) Fluid flow and weld penetration in stationary arc welds. Metall. Transact. $A, \mathbf{1 6}, 203-213$.

6. Knight, C.J. (1979) Theoretical modeling of rapid surface vaporization with back pressure. J. AIAA, 17, 519-523.

Received 10.01.2014 